\title{
Qualidade de vida relacionada com a saúde de pessoas idosas numa perspetiva de género
}

\author{
Fernanda Daniel $^{\mathrm{a}-\mathrm{c}}$ Rosa Monteiro ${ }^{\mathrm{a}, \mathrm{d}}$ Sara Antunes $^{\mathrm{a}}$ Raquel Fernandes $^{\mathrm{e}}$ \\ Pedro Lopes Ferreirab, c, $f$ \\ ${ }^{a}$ Instituto Superior Miguel Torga, Coimbra, Portugal; ${ }^{b}$ Centro de Estudos e Investigação em Saúde da \\ Universidade de Coimbra, Coimbra, Portugal; ' ${ }^{C}$ Centro de Inovação em Biomedicina e Biotecnologia, \\ Coimbra, Portugal; ${ }^{d}$ Centro de Estudos Sociais da Universidade de Coimbra, Coimbra, Portugal; \\ eFaculdade de Medicina da Universidade de Coimbra, Coimbra, Portugal; ${ }^{\mathrm{f}}$ Faculdade de Economia da \\ Universidade de Coimbra, Coimbra, Portugal
}

\section{Palavras chave}

Qualidade de vida · Saúde · Género · Envelhecimento

\section{Resumo}

O objetivo deste estudo foi avaliar a qualidade de vida relacionada com a saúde de pessoas idosas segundo o sexo. Para isso foram inquiridas 300 pessoas idosas com uma média de $74,0 \pm 8,5$ anos. Para a avaliação da qualidade de vida relacionada com a saúde utilizou-se a versão portuguesa do instrumento de medição EQ-5D-3L. As mulheres apresentam piores resultados de qualidade de vida e as associações revelam-se estatisticamente significativas nas dimensões relacionadas com os cuidados pessoais, as atividades habituais, a dor/mal-estar e a ansiedade/depressão. As diferenças no índice de qualidade de vida relacionada com a saúde revelam-se significativas tanto para o sexo como para a idade.

(c) 2018 The Author(s). Published by S. Karger AG, Basel on behalf of NOVA National School of Public Health

\section{Health-Related Quality of Life of Elderly under a Gender Perspective}

\section{Keywords}

Quality of life $\cdot$ Health $\cdot$ Gender $\cdot$ Aging

\section{Abstract}

The objective of this study was to assess health-related quality of life of elderly people under a gender perspective. We surveyed 300 elderlies with an average of $74.0 \pm$ 8.5 years. To assess the health-related quality of life we used the Portuguese version of the EQ-5D-3L measuring instrument. Women always present worse results in terms of quality of life and the associations are statistically significant in the areas of self-care, usual activities, pain/ discomfort, and anxiety/depression. The differences in health-related quality of life are statistically significant for both genders, as well as for age.

C 2018 The Author(s). Published by S. Karger AG, Basel on behalf of NOVA National School of Public Health

\section{KARGER}

E-Mail karger@karger.com www.karger.com/pjp

Karger Open access (c) 2018 The Author(s). Published by S. Karger AG, Basel on behalf of NOVA National School of Public Health

This article is licensed under the Creative Commons AttributionNonCommercial-NoDerivatives 4.0 International License (CC BYNC-ND) (http://www.karger.com/Services/OpenAccessLicense) Usage and distribution for commercial purposes as well as any distribution of modified material requires written permission.
Pedro L. Ferreira, $\mathrm{PhD}$

Faculdade de Economia da Universidade de Coimbra Av Dias da Silva 165

PT-3004-512 Coimbra (Portugal)

E-Mail pedrof@fe.uc.pt; fernanda-daniel@ismt.pt 


\section{Introdução}

Considera-se atualmente que a forma como envelhecemos é extraordinariamente heterogénea, fortemente dissemelhante e eivada de assimetrias várias [1]. Porém, esta heterogeneidade é normalmente escamoteada numa narrativa homogeneizadora que descreve este processo como único, categorizando as pessoas idosas como um grupo. Generalizações excessivas deste processo são, contudo, denunciadas em alguns estudos críticos que evidenciam que o processo de envelhecimento, plástico e diverso, oculta diferenças e desigualdades. Diferenças marcadas pela etnia, idade, sexo e situação socioeconómica concorrem para a diversidade da experiência do envelhecimento $[2,3]$.

No que concerne às diferenças segundo o sexo, estas são o resultado de percursos biográficos calcorreados de forma masculinizada ou feminizada. Este percurso começa com a escolha do nome que nos atribuem que reflete, comumente, o sexo com que nascemos [4]. A educação, os modos de socialização, a carreira profissional, os papéis sociais e as redes extrafamiliares contribuem para que os percursos de vida sejam vivenciados de forma diferente para quem nasce homem ou mulher. Apesar de manifestas evoluções sociais, as desigualdades persistem. Assim, na velhice encontramos desigualdades que refletem, na sua maioria, construções sociais e condições de vida diferenciadas. As relações sociais de género são determinantes das condições de vida e de saúde de homens e mulheres [5]. Papéis sociais e representações diferentes afetam a relação com a doença, assim como com os cuidados de saúde e do corpo. Comportamentos de risco ou protetores para a saúde estão associados a atributos sociais de masculinidade e feminilidade que podem, de acordo com a idade e o contexto, transformar-se numa vantagem ou numa desvantagem [6].

Uma história permeada por estatutos sociais diferentes concorre para desigualdades em saúde. Como referem Crisp et al., o empobrecimento a que foram sujeitos os portugueses no século passado é um fator decisivo na explicação dos problemas de saúde da população, em particular da população mais idosa [7]. Portugal plasma nas camas dos hospitais o impacte negativo das desigualdades de acesso a bens de primeira necessidade a que muitas das pessoas idosas de hoje estiveram sujeitas na infância e que se reflete na prevalência de determinados tipos de cancro, e.g., intestino e estômago. No que concerne às mulheres, estas apresentam maior esperança de vida que os seus congéneres masculinos; contudo, o número de anos que podem esperar viver sem limitações funcionais ou inca- pacidade é inferior em termos absolutos ao dos homens no cenário português e, em termos proporcionais, ao nível europeu [8]. Evidências indicam que as desigualdades na saúde são apenas uma consequência de um sistema social que potencia desigualdades [9].

Segundo Sen, George e Östlin, o não reconhecimento das desigualdades de género resulta da resistência fundada na ideia de que as diferenças são de ordem biológica e não o resultado das relações sociais de género [10]. Contrariando esta tendência, Doyal defende a necessidade de identificar as ligações entre sexo e género para clarificar as relações de ambos com os determinantes da saúde [11]. É um facto que determinadas características biológicas poderão, só por si, explicar diferenças no perfil sanitário experienciado pelos homens e pelas mulheres. Algumas das diferenças entre os padrões de mortalidade e de morbilidade entre os sexos resulta da influência de fatores biológicos como é o caso do cancro do colo do útero e do cancro da próstata. Esta evidência que resulta de diferenças entre sexos, nomeadamente ao nível genético, hormonal e metabólico, não deve contudo excluir o reconhecimento de que as diferenças entre os sexos ultrapassam esses domínios [12]. Segundo alguns autores [9], torna-se, portanto, fundamental e cada vez mais consensual, que se tem de adotar uma perspetiva de género na saúde, distinguindo o que é consequência de fatores biológicos e o que é resultado do impacte das desigualdades de género. Isto porque o género influencia as experiências de saúde ou doença de mulheres e homens e as vulnerabilidades respetivas, impregna também a organização dos sistemas de saúde e o desempenho de profissionais [13]. Também Esteban estatuiu que as mulheres e os homens apresentam diferenças muito significativas no que diz respeito a hábitos de saúde, esperança de vida, modos de adoecer e morrer, auto perceção da sua saúde e utilização dos serviços de saúde [5].

O presente estudo procurou captar como e onde é que estas especificidades se concretizam em diferenças, avaliando a forma como os homens e as mulheres da amostra pontuam no que respeita aos fatores sanitários captados pela versão portuguesa do instrumento de medição de qualidade de vida EuroQol EQ-5D-3L.

\section{Métodos}

Realizou-se um estudo quantitativo e transversal. Para a sua concretização utilizou-se como critérios de seleção da amostra a idade - população de um concelho português com idade igual ou superior a 60 anos - e a ausência de deterioração cognitiva. $O$ ponto de corte, utilizado para a idade, foi o adotado pelas Nações Uni-
60

Port J Public Health 2018;36:59-65 DOI: $10.1159 / 000490929$
Daniel/Monteiro/Antunes/Fernandes/ Ferreira 
das nos indicadores de envelhecimento. Aliás, neste estudo, a análise apenas considerou as variáveis sexo e idade.

A população do Concelho da Lousã, segundo os Censos de 2011, é constituída por 4.157 indivíduos com idade superior ou igual a 60 anos. No caso presente trabalhou-se com um nível de confiança de $99 \%$ e um erro de $5 \%$. Assim, a amostra implicou a inquirição de 300 indivíduos provenientes da lista das pessoas idosas portadores do Cartão Municipal Sénior e da população que frequenta respostas sociais. Os portadores do Cartão Municipal Sénior foram informados sobre o estudo, através de circular redigida pelo Presidente do Município. Tendo em conta que a investigação decorreu no âmbito da rede social concelhia, foi solicitado aos parceiros, nomeadamente aos das Instituições Particulares de Solidariedade Social, para administrarem o questionário aos usuários das respetivas instituições.

$\mathrm{Na}$ recolha de dados, utilizou-se a versão portuguesa do EQ-5D-3L que mede a qualidade de vida relacionada com a saúde percecionada pelos indivíduos. As cinco dimensões que o compõem (mobilidade, cuidados pessoais, atividades habituais, dor/ mal-estar e ansiedade/depressão) permitem gerar um índice que representa a intensidade da preferência por um determinado estado de saúde. O cálculo do índice é efetuado com base nos coeficientes descritivos e numa regressão do modelo desenvolvido a partir do sistema de valores da população portuguesa e de uma amostra significativa da população portuguesa por idade e sexo $[14,15]$. O EQ-5D-3L é um instrumento suficientemente curto para ser utilizado em conjunto com outras medidas, demorando aproximadamente um minuto para ser preenchido. Cada uma das cinco dimensões tem três níveis de gravidade, correspondendo o nível 1 a "não tenho problemas...," o nível 2 a "tenho alguns problemas..." e o nível 3 "tenho problemas extremos." Assim sendo, este sistema permite descrever um total de $3^{5}=243$ estados de saúde distintos [16].

A aplicação do questionário foi efetuada individualmente em forma de entrevista presencial. Com o objetivo de respeitar os procedimentos éticos previstos nas pesquisas com seres humanos exigidos pela Declaração de Helsínquia, o questionário apresentava, além de uma declaração de consentimento informado e esclarecido, os objetivos e a utilidade do estudo. Os inquiridos autorizaram a recolha de dados, tendo sido garantido o anonimato e a confidencialidade dos dados. Os espaços onde decorreu a administração apresentavam condições de privacidade.

O programa informático utilizado para efetuar a análise estatística foi o IBM Statistical Package for Social Sciences (SPSS v.20). $\mathrm{Na}$ análise dos dados utilizou-se a estatística descritiva e inferencial e na descrição univariada recorreu-se a medidas de tendência central e de dispersão. Por outro lado, o teste de ajustamento do qui-quadrado foi utilizado para verificar se a distribuição das frequências observadas dos dados se ajustava a um modelo teórico pré-determinado, no caso presente, à população do município da Lousã. O teste do qui-quadrado de independência foi utilizado para verificar a existência de associações entre cada uma das cinco dimensões com o sexo. Também o teste $t$ de Student para grupos independentes foi utilizado para detetar diferenças entre médias no índice de qualidade de vida e, para determinar diferenças na qualidade de vida a partir de duas variáveis categóricas (grupos etários e sexo), utilizou-se a ANOVA de duas vias. Os grupos etários foram constituídos com base nos cortes etários utilizados pelo grupo EuroQoL e permitiram a constituição de três grupos etários. O primeiro grupo foi constituído por pessoas com idades até os 69
Table 1. Indicadores descritivos da amostra e da população com idade $\geq 60$ anos do concelho da Lousã

\begin{tabular}{lccccc}
\hline Variável & \multicolumn{2}{c}{ Amostra } & & \multicolumn{2}{c}{ Lousã } \\
\cline { 2 - 3 } \cline { 5 - 6 } & $n$ & $\%$ & & \\
& & & & \\
\hline Sexo & 200 & 66,7 & & 2.318 & 55,8 \\
$\quad$ Feminino & 100 & 33,3 & & 1.839 & 44,2 \\
$\quad$ Masculino & & & & \\
Idade & 112 & 37,3 & & 1.900 & 45,7 \\
$\quad 60$ a 69 anos & 101 & 33,7 & & 1.501 & 36,1 \\
70 a 79 anos & 87 & 29,0 & & 756 & 18,2 \\
$\quad 80$ ou mais anos & $74,0 \pm 8,5$ & & & \\
$\quad$ Média \pm desvio-padrão & $60-100$ & & & \\
$\quad$ Min-máx & & & & \\
\hline
\end{tabular}

anos, o segundo com idades compreendidas entre os 70 e os 79 anos e o último agregou as pessoas com 80 e mais anos de idade. Por fim, o valor de eta quadrado parcial foi apresentado como medida do tamanho do efeito e definiu-se para o estudo um nível de significância de $5 \%$.

\section{Resultados}

Trabalhou-se com uma amostra composta por 300 indivíduos, com 60 e mais anos de idade. A idade média dos participantes situou-se nos 74,0 anos $(D P=8,5)$ e a sua composição refletiu a acentuada expressão numérica das mulheres nas idades avançadas. A tabela 1 apresenta as principais características descritivas da amostra e da população em estudo.

A associação entre as cinco dimensões do EQ-5D-3L e o sexo, assim como as diferenças na avaliação compósita do EQ-5D para os homens e para as mulheres estão apresentadas na tabela 2 .

Analisando esta tabela verificou-se que a associação entre as dimensões e o sexo é estatisticamente significativa no domínio dos cuidados pessoais, das atividades habituais, da dor/mal-estar e da ansiedade/depressão. No que concerne ao índice EQ-5D verificaram-se diferenças estatisticamente significativas quando comparamos as pontuações dos homens e das mulheres. Em relação aos homens, as mulheres apresentam praticamente sempre valores correspondentes a uma maior gravidade da doença e uma pior perceção da qualidade de vida.

Foi também efetuada uma Anova de duas vias para explorar os efeitos principais do sexo e da idade na qualidade de vida relacionada com a saúde. Os inquiridos, categorizados em três grupos etários, apresentaram uma in- 
Table 2. Comparação dos valores de qualidade de vida segundo o "sexo"

\begin{tabular}{|c|c|c|c|c|c|c|}
\hline & \multicolumn{2}{|c|}{ Feminino } & \multicolumn{2}{|c|}{ Masculino } & \multirow[t]{2}{*}{$\chi^{2}$} & \multirow[t]{2}{*}{$p$} \\
\hline & $n$ & $\%$ & $n$ & $\%$ & & \\
\hline \multicolumn{5}{|l|}{ Mobilidade } & \multirow[t]{3}{*}{0,64} & \multirow[t]{3}{*}{0,422} \\
\hline Sem problemas & 147 & 73,7 & 77 & 77,8 & & \\
\hline Com problemas & 53 & 26,5 & 22 & 22,2 & & \\
\hline \multicolumn{5}{|l|}{ Cuidados pessoais } & \multirow[t]{3}{*}{6,50} & \multirow[t]{3}{*}{0,011} \\
\hline Sem problemas & 167 & 83,5 & 94 & 94,0 & & \\
\hline Com problemas & 33 & 16,5 & 6 & 6,0 & & \\
\hline \multicolumn{5}{|l|}{ Atividades habituais } & \multirow[t]{3}{*}{11,24} & \multirow[t]{3}{*}{0,001} \\
\hline Sem problemas & 152 & 76,0 & 92 & 92,0 & & \\
\hline Com problemas & 48 & 24,0 & 8 & 8,0 & & \\
\hline \multicolumn{5}{|l|}{ Dor/mal-estar } & \multirow[t]{3}{*}{7,29} & \multirow[t]{3}{*}{0,007} \\
\hline Sem problemas & 83 & 41,5 & 58 & 58,0 & & \\
\hline Com problemas & 117 & 58,5 & 42 & 42,0 & & \\
\hline \multicolumn{7}{|l|}{ Ansiedade/depressão } \\
\hline Sem problemas & 80 & 40,0 & 56 & 56,0 & \multirow[t]{2}{*}{6,89} & \multirow[t]{2}{*}{0,009} \\
\hline \multirow[t]{2}{*}{ Com problemas } & 120 & 60,0 & 44 & 44,0 & & \\
\hline & $M$ & $D P$ & $M$ & $D P$ & $|t|$ & $p$ \\
\hline Índice - EQ-5D & 0,60 & 0,29 & 0,74 & 0,24 & 4,42 & $<0,001$ \\
\hline
\end{tabular}

M, média; DP, desvio-padrão; $|t|$, teste $t$ de Student para grupos independentes, valor absoluto; $\chi^{2}$, qui quadrado; $p$, significância, $p<0,05$.

teração com o género não estatisticamente significativa $\left(F=1,0 ; p=0,368 ; \eta_{\mathrm{p}}^{2}=0,007\right)$. Existem, contudo, alguns efeitos principais a reportar relativamente aos grupos etários $\left(F=8,4 ; p>0,001 ; \eta_{\mathrm{p}}{ }^{2}=0,054\right)$ e ao sexo $(F=18,0$; $\left.p>0,001 ; \eta_{\mathrm{p}}^{2}=0,058\right)$. As comparações post-hoc, usando o teste Tukey HSD, revelaram que as pontuações médias do grupo com idades compreendidas entre os 60-69 anos $(M=0,70 ; D P=0,24)$ eram estatisticamente diferentes das do grupo com 80 ou mais anos de idade $(M=0,64 ; D P=$ $0,29)$ e que o grupo com idades compreendidas entre os 70 e os 79 anos de idade $(M=0,67 ; p=0,30)$ era estatisticamente diferente do grupo com 80 ou mais anos de idade $(M=0,64 ; D P=0,29)$, conforme $\mathrm{o}$ apresentado na figura 1 .

\section{Discussão}

A amostra evidenciou uma relação de quase dois terços de mulheres, o que vai, praticamente, ao encontro das estatísticas do município da Lousã que refletem uma feminização da população com idade igual ou superior a 60 anos, mas com uma proporção ligeiramente inferior, conforme se pode observar na tabela 1 .
O mesmo acontece relativamente aos agrupamentos etários da amostra. Se compararmos a nossa proporção de pessoas nos diferentes agrupamentos etários com os da população da mesma idade do município da Lousã verifica-se que as proporções decrescem com a idade. Contudo, existem diferenças significativas nas proporções $\left(\chi^{2}=\right.$ 24,39; $p<0,001)$ entre os três grupos etários.

$\mathrm{Na}$ nossa amostra encontraram-se diferenças estatisticamente significativas em quatro das cinco dimensões da qualidade de vida medidas pela componente descritiva do EQ-5D-3L. As dimensões referentes aos cuidados pessoais $\mathrm{e}$ às atividades habituais dizem, respetivamente, respeito às componentes básicas e instrumentais das atividades de vida diária. A capacidade de cuidar de si e do seu ambiente, geralmente também denominada atividade de vida diária, constitui um fator crítico para a saúde e bem-estar das pessoas idosas, como aliás é confirmado por alguns autores [17]. De facto, é de esperar que a perda de capacidades funcionais afete a realização das atividades diárias e potencie sentimentos de maior esforço na sua realização, com impactes ao nível pessoal, social e económico. Estudos reportam diferenças entre os sexos, tal como também aqui se encontraram $[18,19]$. Tentando explicar
Daniel/Monteiro/Antunes/Fernandes/ Ferreira 


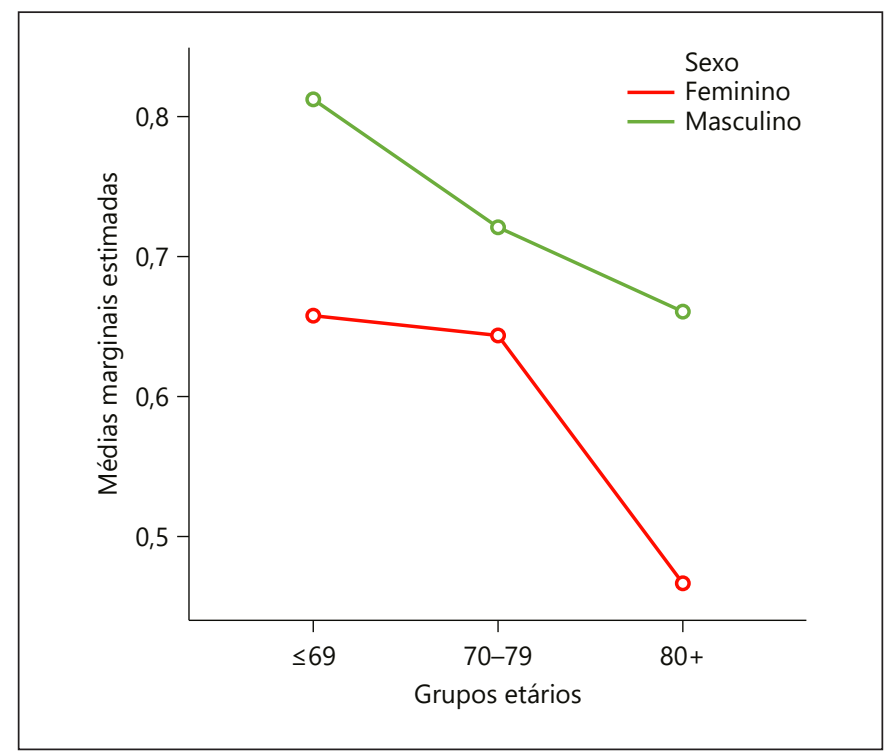

Fig. 1. EQ-5D segundo os grupos etários e o sexo.

este fenómeno podemos acrescentar que a maior incapacidade nas mulheres está, provavelmente, associada ao facto de apresentarem maior prevalência de condições incapacitantes não fatais e, com isto, uma sobrevivência maior, tornando-se mais suscetíveis ao desfecho em questão.

A dimensão dor/mal-estar é também, nos nossos resultados, melhor para os homens do que para as mulheres. Como tem sido referido ao longo deste trabalho, embora as mulheres tenham uma maior longevidade, isso não significa que a qualidade de vida as acompanhe. As doenças crónicas surgem mais cedo do que nos homens, o que dificulta o seu desempenho diário. Não obstante terem ganho sete anos de vida relativamente aos homens, a verdade é que as mulheres sofrem mais doenças crónicas e incapacitantes, o que limita a sua capacidade para uma vida diária autónoma e amplifica a necessidade de institucionalização [20].

Dados nacionais indicam que, apesar de as mulheres apresentarem maior esperança de vida que os seus congéneres masculinos, a esperança de vida ajustada a deficiências é inferior em Portugal (6,6 anos) quando comparada com a média da UE (8,4 anos). Isto apesar de as mulheres sofrerem de incapacidade numa fase mais prematura. Evidências parecem indicar que as desigualdades na saúde são apenas uma consequência de um sistema social que potencia desigualdades [9]. De facto, homens e mulheres, ocupam diferentes posições na estrutura social que medeiam a sua exposição a riscos que são prejudiciais à saú-

Qualidade de vida relacionada com a saúde de pessoas idosas de, a sua participação em comportamentos prejudiciais à saúde, e seu acesso a bens e recursos que promovem o bem-estar [21].

A dimensão ansiedade/depressão apresenta, também, diferenças significativas quando se comparam a pontuação dos homens com a das mulheres. Este resultado acompanha os obtidos em outros estudos que indicam que o sexo (feminino), as doenças somáticas, o declínio cognitivo e funcional, a falta ou perda de contato social e a história de depressão anterior são os principais indicadores envolvidos nos distúrbios e sintomas depressivos [22]. No mesmo sentido vai o relatório sobre a saúde no mundo da Organização Mundial de Saúde quando afirma que os transtornos da ansiedade e a depressão são mais prevalentes na população feminina [23]. A evidência tem demonstrado diferenças ao nível da saúde mental quando se comparam homens e mulheres, mostrando através de indicadores psicossociais, que o género é uma construção psicossocial que influencia inevitavelmente a expressão da saúde mental $[24,25]$. Das várias causas apontadas, surge a explicação de que as mulheres têm vindo a ser socializadas no sentido de internalizar o distress, o que contribui para desordens associadas à depressão, ansiedade e ideação suicida. Por seu lado, os homens têm vindo a ser encorajados a agir e a expressar o seu distress [24].

Além disto, a saúde assume um papel central nas idades avançadas, existindo determinantes sociais e económicos que condicionam tanto a saúde dos homens como a das mulheres [25]. De facto, as desigualdades socioeconómicas expressam-se na velhice, com as pessoas idosas mais desfavorecidas a requererem ajuda da parte do sistema de apoio social de forma a lidarem com os problemas [26]. Por outro lado, a degradação da qualidade de vida e das condições de saúde aumenta em ambos os sexos, com o aumento da idade. Porém, essa degradação é mais acentuada no caso das mulheres, conforme o ilustrado na figura 1 onde podemos observar que, com o aumento da idade, a tendência da qualidade de vida é decrescente, mas a queda mais abrupta dá-se no caso das mulheres, na passagem do grupo dos 70-79 anos, para o grupo das que têm 80 anos ou mais.

Sabe-se também que as coortes que hoje constituem a população idosa foram socializadas numa cultura impregnada de assimetrias entre os sexos, consagradas inclusivamente na legislação do Estado Novo [27]. Assim, no envelhecimento, parecem salientar-se diferenças significativas entre grupos sociais e fatores que atuam de forma conjugada. As mulheres geralmente acumulam fatores de desigualdade motivados pelas relações sociais de género, que as tornam particularmente vulneráveis. São 
elas quem têm carreiras contributivas mais fragmentadas ou mesmo inconsistentes, o que determina gaps nas pensões que chegam a $40 \%$ das dos homens, por exemplo. A título de exemplo, em 2011, a pensão média das mulheres portuguesas que tinham trabalhado no sector privado correspondia a $57,2 \%$ da dos homens (estatísticas da segurança social, outubro de 2011).

Existem algumas limitações que devem ser levadas em conta. Embora sabendo que muitas outras variáveis sociodemográficas estão estritamente relacionadas com a idade e com o sexo, a utilização apenas destas duas variáveis explicativas da qualidade de vida pode ser considerada uma limitação. Apesar disto, estamos convencidos que a inclusão de outras variáveis iria reforçar as conclusões a que este estudo chegou.

Por outro lado, os seus participantes foram selecionados de entre os idosos portadores do Cartão Municipal Sénior e de entre a população que frequenta respostas sociais. Esta forma de seleção pode eventualmente ter criado enviesamentos que limitem a generalização das conclusões.

Não obstante estas ressalvas, consideramos que para além da variabilidade intraindividual do processo de envelhecimento, há a destacar a variabilidade interindividual, uma vez que vivências diferentes, a par de papéis de género diferentes, concorrem para a heterogeneidade do envelhecimento. Assim, consideramos importante dar conta destas diferenças para que as respostas sociais possam respeitar as necessidades e as diferenças de cada sujeito. Parece-nos também importante estimular um novo planeamento organizacional potenciador de uma vida ativa e participativa, permitindo que as pessoas idosas se sintam parte integrante das decisões tomadas, não só no que respeita a si próprias como também no que concerne ao meio que as envolve.

Pretendeu-se, com este trabalho, contribuir para uma análise com perspetiva de género da qualidade de vida e do bem-estar no envelhecimento, contrariando a invisibilidade a que tem sido votada a especificidade das mulheres idosas e de meia-idade [28], até mesmo pelas análises feministas [29-31].

Com efeito, investigações realizadas a partir de estudos de coorte, têm analisado as diferenças entre os homens e as mulheres, chamando a atenção para a ausência de visibilidade das mulheres idosas e das suas desvantagens cumulativas [28]. Outros estudos [2] apontam os impactes diferenciadores das vivências de género sobre a geração mais velha, que vive em regimes de género mais tradicionalistas, onde os papéis e as assimetrias entre mulheres e homens eram mais rígidos e clivados, nomeadamente ao nível do exercício da autoridade dos homens sobre as mulheres e os filhos no seio das famílias. A população idosa vivenciou uma assimetria relacional, que marcou percursos, trajetórias e identidades que pode determinar a vivência da velhice para o sujeito e nas suas relações. Este olhar sexualizado permite-nos dar conta de processos através dos quais um fenómeno (identidade, instituição, linguagem ou processo) passa a integrar explicitamente um significado associado às relações sociais entre mulheres e homens [32].

\section{References}

1 Fonseca AM: Desenvolvimento humano e envelhecimento. Lisboa: Climepsi Editores; 2005.

2 Daniel F, Simões T, Monteiro R: Representações sociais do «envelhecer no masculino»e do «envelhecer no feminino». Ex aequo 2012; 26:13-26.

3 Daniel F: Sete mulheres para cada homem: uma análise de relações de masculinidade. População e Sociedade 2011;19:157-167.

4 Shaffer D: Social and personality development. Pacific Grove, CA: Brooks/Cole; 1994.

5 Esteban ML: El estudio de la salud y el género: las ventajas de un enfoque antropológico y feminista. Salud Colect 2006;2:9-20.

6 Saleiro S, Ferreira V, Monteiro R, Lopes M, Múrias C: Guia para a integração a nível local da perspetiva de género na saúde e ação social. Coimbra: Centro de Estudos Sociais da Universidade de Coimbra; 2016.
7 Crisp N, Berwick D, Kickbusch I, Bos W, Antunes JL, Barros PP, et al: Um futuro para a saúde: todos temos um papel a desempenhar. Lisboa: Fundação Calouste Gulbenkian; 2014.

8 OECD. Health at a glance 2015: OECD indicators. Paris: OECD Publishing; 2015.

9 Denton M, Prus S, Walters V: Gender differences in health: a Canadian study of the psychosocial, structural and behavioural determinants of health. Soc Sci Med 2004;58:25852600.

10 Sen G, George A, Östlin P: Engendering international health: the challenge of equity. Cambridge, MA: MIT Press; 2002.

11 Doyal L: Gender and the 10/90 gap in health research. Bull World Health Organ 2004;82: 162.

12 Doyal L: Sex, gender, and health: the need for a new approach. BMJ 2001;323:1061-1063.
13 Theobald S, Tolhurst R, Elsey H, Standing H: Engendering the bureaucracy? Challenges and opportunities for mainstreaming gender in Ministries of Health under sector-wide approaches. Health Policy Plan 2005;20:141149.

14 Ferreira LN, Ferreira PL, Pereira LN, Oppe M: EQ-5D Portuguese population norms. Qual Life Res 2014;23:425-430.

15 Ferreira LN, Ferreira PL, Pereira LN, Oppe M: The valuation of the EQ-5D in Portugal. Qual Life Res 2014;23:413-423.

16 Ferreira PL, Ferreira LN, Pereira LN: Contributos para a validação da versão portuguesa do EQ-5D. Acta Med Port 2013;26:664-675.

17 Fillenbaum GG: The wellbeing of the elderly: approaches to multidimensional assessment. WHO Offset Publ 1984;1-99.

18 INE. Saúde e incapacidades em Portugal 2011. Lisboa: Instituto Nacional de Estatística; 2012.

Daniel/Monteiro/Antunes/Fernandes/ Ferreira
64

Port J Public Health 2018;36:59-65

DOI: $10.1159 / 000490929$ 
19 INE. Esperanças de vida sem incapacidades físicas de longa duração. Lisboa: Instituto $\mathrm{Na}$ cional de Estatística; 2000.

20 Martins R, Barbosa D, Júnior J, Oliveira Y: Feminização da velhice: caracterização de seu perfil no contexto asilar. João Pessoa, Paraíba: Universidade Federal da Paraíba; 2007. (cited 12.02.2016). Available from: http://itaporanga.net/genero/1/GT11/18.pdf.

21 Bird CE, Rieker PP: Gender matters: an integrated model for understanding men's and women's health. Soc Sci Med 1999;48:745755.

22 Irigaray TQ, Schneider RH: Prevalência de depressão em idosas participantes da Universidade para a Terceira Idade. Rev Psiquiatr Rio Gd Sul 2007;29:19-27.
23 OMS. Relatório sobre a saúde no mundo 2001: saúde mental: nova concepção, nova esperança. Genebra: OPAS. OMS; 2001.

24 Rabasquinho C, Pereira H: Género e saúde mental: uma abordagem epidemiológica. Aná Psicológica 2007;25:439-454.

25 WHO. Active ageing: a policy framework. Geneva: World Health Organization; 2002.

26 Lloyd L, Tanner D, Milne A, Rayd M, Richards S, Sullivan MP, et al: Look after yourself: active ageing, individual responsibility and the decline of social work with older people in the UK. Eur J Soc Work 2013;17:322-335.

27 Monteiro R: Feminismo de estado emergente na transição democrática em Portugal na década de 1970. Dados 2013;56:841-865.
28 Goldani AM: Mulheres e envelhecimento: desafios para novos contratos intergeracionais e de gênero. In: Camarano AA, editor. Muito além dos 60:os novos idosos brasileiros. Rio de Janeiro: Instituto de Pesquisa Economica Aplicada; 1999, pp 75-113.

29 Arber S, Ginn J: Relación entre género y envejecimiento: enfoque sociológico. Madrid: Narcea; 1996.

30 Miller A, Simeth A: Combating invisibility: older women stereotypes revised. Oshkosh Sch 2007;2:49-56.

31 Wheeler HR: Women and aging: a guide to the literature. Boulder, CO: Lynne Rienner Publishers; 1997.

32 Stetson DM, Mazur A: Comparative state feminism. Thousand Oaks, CA: SAGE Publications; 2005. 